\title{
Baseline Elevated Serum Angiopoietin-2 Predicts Long-Term Non-Regression of Liver Fibrosis After Direct-Acting Antiviral Therapy for Hepatitis C
}

Goki Suda ( $\sim$ gsudgast@pop.med.hokudai.ac.jp)

Hokkaido University

Naoki Kawagishi

Hokkaido University

Megumi Kimura

Hokkaido University

Osamu Maehara

Hokkaido University

Ren Yamada

Hokkaido University

Yoshimasa Tokuchi

Hokkaido University

Akinori Kubo

Hokkaido University

Takashi Kitagataya

Hokkaido University

Taku Shigesawa

Hokkaido University

Kazuharu Suzuki

Hokkaido University

Masatsugu Ohara

Hokkaido University

Masato Nakai

Hokkaido University

Takuya Sho

Hokkaido University

Mitsuteru Natsuizaka

Hokkaido University

Kenichi Morikawa

Hokkaido University

Koji Ogawa 
Hokkaido University

\section{Yusuke Kudo}

Hokkaido University

Mutsumi Nishida

Hokkaido University

Naoya Sakamoto

Hokkaido University

\section{Research Article}

Keywords: hepatitis C virus, direct-acting antiviral agent, angiopoietin-2, liver stiffness measurement

Posted Date: January 14th, 2021

DOl: https://doi.org/10.21203/rs.3.rs-143428/v1

License: (c) (i) This work is licensed under a Creative Commons Attribution 4.0 International License. Read Full License

Version of Record: A version of this preprint was published at Scientific Reports on April 28th, 2021. See the published version at https://doi.org/10.1038/s41598-021-88632-7. 


\section{Abstract}

We previously revealed that Angiopoietin-2 (Ang2) predicts non-regression of liver fibrosis based on liver stiffness measurement (LSM) at 24 weeks after anti-hepatitis C virus (HCV) treatment. In this study, we extended the observational period to 96 weeks to investigate the factors associated with non-regression after treatment with direct-acting-antivirals (DAAs). Patients treated with DAAs who underwent transient elastography at baseline and 24 and 96 weeks after DAA therapy were included. Baseline and posttreatment serum Ang2 levels were measured. Liver fibrosis stages were defined based on LSM. Multivariate regression was used to evaluate factors associated with non-regression of liver fibrosis between various time points. In total, 110 patients were included. Of these, $11 \%$ showed non-regression of LSM-based fibrosis stage at 96 weeks after DAA therapy. In multivariate analysis, advanced liver fibrosis stage and high baseline Ang2 levels were significantly associated with non-regression at 96 weeks. In patients with advanced liver fibrosis (F3/4), baseline Ang2 levels were associated with non-regression of liver fibrosis stage. Between SVR24 and SVR96, post-treatment Ang2 levels and controlled attenuation parameter values at SVR24 were significantly associated with non-regression of liver fibrosis stage in patients with F3/4. Thus, serum Ang2 levels are an important target for monitoring and therapy.

\section{Introduction}

Direct-acting antiviral agents (DAAs) targeting hepatitis $\mathrm{C}$ virus (HCV) proteins, including HCV protease, HCV NS5A, and HCV polymerase, have revolutionized anti-HCV therapy. Various clinical trials and realworld clinical studies have shown that DAA therapy for patients with HCV infection, including those previously classified as difficult-to-treat in the interferon treatment era, could achieve a sustained viral response (SVR) rate exceeding 95\%. ${ }^{1-10}$ Most patients who achieve SVR experience an improvement in liver fibrosis ${ }^{11,12}$ with a low rate of hepatocellular carcinoma (HCC) occurrence. However, even after successful HCV eradication by IFN or DAAs, some patients develop HCC and/or the deterioration of liver fibrosis. ${ }^{11-14}$ Importantly, Tachi et al. showed that the deterioration of liver fibrosis after HCV eradication is significantly associated with HCC occurrence. ${ }^{11}$ Thus, predicting changes in liver fibrosis after HCV eradication is a clinically important issue.

Angiopoietin-2 (Ang2) has an antagonistic effect on Tie2-mediated signalling, which is associated with vessel stabilization in some situations. ${ }^{15,16}$ We and other groups have shown that serum Ang2 levels are elevated in patients with advanced liver fibrosis and advanced $\mathrm{HCC}, 13,17,18$ and this increase is partially due to a portal hypertension-induced decrease in blood flow. ${ }^{19,20}$ Thus, we hypothesized that portal hypertension-induced increases in serum Ang2 cause vascular leakage and inflammation, resulting in the deterioration of liver fibrosis. Consistent with this hypothesis, Seko et al. recently reported that the presence of portal hypertension-induced varices is an independent predictor of the deterioration of liver fibrosis based on the Fibrosis-4 (FIB-4) index at 96 weeks after the end of DAA treatment. ${ }^{21}$ In addition, we recently reported the possibility that baseline serum Ang2 levels could predict non-regression of liver fibrosis stage based on liver stiffness measurement (LSM) at 24 weeks after DAA therapy with high 
accuracy (sensitivity 0.882 , specificity 0.733 ). ${ }^{13}$ However, in this previous study, the observational period was relatively limited.

In this study, we aimed to investigate the factors associated with non-regression of liver fibrosis based on LSM at 96 weeks after DAA completion and to validate the predictive value of baseline Ang2 levels over a longer observational period.

\section{Methods}

\section{Patients and study design}

A total of 208 HCV-infected patients at Hokkaido University Hospital between October 2014 and July 2016 were screened. The following inclusion criteria were applied: treated with IFN-free DAAs between October 2014 and July 2016, achieved SVR at 24 weeks after treatment completion (SVR24), had complete clinical information, had preserved serum samples at baseline and post-treatment (end of treatment to SVR24), and had FibroScan data for LSM at baseline, SVR24, and SVR96. Patients were excluded if they could not achieve SVR, had missing clinical information or FibroScan examination at baseline, SVR24, and SVR96, had a lack of serum samples at baseline and/or at post-treatment, had another liver disease or were receiving haemodialysis, and/or had a history of HCC.

Included patients were typically evaluated by the attending physician every 2 weeks during the treatment period and every 3 months after treatment completion. Data were collected at baseline, SVR24, and SVR96, including clinical information (laboratory data, HCV data, and treatment history of hypertension, diabetes mellitus, and dyslipidaemia) and FibroScan examination.

Preserved serum samples were used to measure serum Ang2 levels by a commercial enzyme-linked immunosorbent assay according to the manufacturer's protocol (R\&D Systems, Minneapolis, MN, USA). ${ }^{13}$

This study conformed to the ethical guidelines of the Declaration of Helsinki and was approved by the ethics committee of Hokkaido University Hospital (UMIN000031091).

All enrolled patients provided written informed consent to participate in this study.

\section{Definition of liver fibrosis stage and regression after successful HCV eradication}

FibroScan 502 (Echosens, Paris, France) was used for LSM and controlled attenuation parameter (CAP) evaluation with the M-probe and XL-probe. As described previously, patients were placed in the supine position with the right hand at the most abducted position during the FibroScan examination

procedure. ${ }^{13,22}$ Similar to our previous report, at least 10 valid measurements were obtained, and effective measurements were defined as those with a success rate of $>60 \%$ and an interquartile range of $<30 \%$. ${ }^{13,22}$

The fibrosis stage was defined according to transient elastography data (FibroScan; Echosens) as described previously, with cut-off values of $7.1 \mathrm{kPa}$ for $\mathrm{F} \geq 2,9.5 \mathrm{kPa}$ for $\mathrm{F} \geq 3$, and $12.5 \mathrm{kPa}$ for $\mathrm{F} 4$. $^{13,23}$ 
In addition, according to a previous report, regression of liver fibrosis was defined as follows: in patients with liver fibrosis stage F2 to F4, the liver fibrosis stage decreased by more than 1 stage after DAA therapy; in patients with liver fibrosis F0/1, the liver fibrosis stage did not deteriorate. ${ }^{13,23}$

\section{Statistical analyses}

Continuous variables were analysed by the paired Mann-Whitney U-test, and categorical data were analysed by the chi-squared test. A multivariate logistic regression analysis with stepwise forward selection was performed with variables identified as significant at $P \leq 0.005$ in univariate analyses of the factors associated with non-regression of liver fibrosis between baseline and at SVR96 and identified as significant at $P \leq 0.001$ in univariate analyses of the factors associated with non-regression of liver fibrosis between SVR24 and SVR96. All $P$-values were two-tailed, and $P<0.05$ was defined as statistically significant. Statistical analyses were performed using SPSS version 24.0 (IBM Japan, Tokyo, Japan).

\section{Results}

\section{Baseline patient characteristics}

A total of 208 patients with HCV infection who received IFN-free DAA therapy between October 2014 and July 2016 and were regularly followed-up at Hokkaido University Hospital were screened. Of these, 110 patients with all FibroScan examination data at baseline, SVR24, and SVR96, complete clinical information, and preserved serum samples obtained at baseline and after treatment were included in this study (Figure S1). Table 1 shows the baseline characteristics of these 110 patients and a comparison of LSM-based liver fibrosis stages F0-2 and F3-4. The median age of patients was 66 years (range, 22-87 years), and 69 patients (62.7\%) were female. The baseline median aspartate aminotransferase (AST) and alanine aminotransferase (ALT) levels were $39 \mathrm{IU} / \mathrm{L}$ (range, 16-180) and $38 \mathrm{IU} / \mathrm{L}$ (range, 6-273), respectively, and the median platelet count was $16.2 \times 10^{4} / \mu \mathrm{L}$ (range, $2.6-37.3 \times 10^{4} / \mu \mathrm{L}$ ).

In total, $19,47,39$, and 5 patients were treated with daclatasvir plus asunaprevir, sofosbuvir plus ledipasvir, sofosbuvir plus ribavirin, and ombitasvir plus paritaprevir, respectively. Additionally, 62, 19, 8, and 21 patients had liver fibrosis stages based on LSM of F0-1, F2, F3, and F4, respectively.

\section{Rate of non-regression at 96 weeks after successful HCV eradication by DAA therapy and associated factors}

Figure 1 summarizes the changes in LSM-based liver fibrosis stage at 96 weeks after successful HCV eradication by DAA therapy. As the baseline liver fibrosis stage increased, the rate of non-regression at 96 weeks after DAA therapy increased. Among patients with baseline F4 stage, 33\% (7/21) showed nonregression based on LSM, whereas $11 \%$ of all patients (12/110) showed non-regression based on LSM at 96 weeks after DAA therapy. 
We further analysed the baseline predictive factors associated with non-regression of liver fibrosis at 96 weeks after DAA initiation. Table 2 provides a comparison of various factors in patients with or without non-regression of liver fibrosis stage based on LSM at 96 weeks after DAA initiation. A univariate analysis revealed that baseline liver fibrosis stage (F0-2 vs. $3-4, P=0.002)$, FIB-4 index $(P=0.005)$, angiopoietin$2(P=0.004)$, HCV-RNA $(P=0.025)$, and AST $(P=0.029)$ were significantly associated with nonregression at 96 weeks after DAA initiation. Subsequently, we included significant factors identified in the univariate analysis $(P \leq 0.005)$ in a multivariate logistic regression analysis (i.e., the liver fibrosis stage, FIB-4 index, and Ang2 levels). As shown in Table 2, the multivariate logistic regression analysis revealed that baseline fibrosis stage (odds ratio 4.56, 95\% confidence interval, 1.13-18.3; $P=0.033$ ) and Ang2 level (odds ratio $1.004,95 \%$ confidence interval, $1.00-1.01 ; P=0.039$ ) were significantly associated with non-regression of liver fibrosis stage based on LSM at 96 weeks after DAA initiation.

Table 3 summarizes the results of a subgroup analysis of patients with baseline fibrosis stage of F3/4. In this group, high Ang2 at baseline was significantly associated with non-regression of liver fibrosis stage based on LSM at 96 weeks after DAA initiation $(P=0.024)$.

\section{Rate of non-regression of LSM-based liver fibrosis stage between 24 and 96 weeks after successful HCV eradication and associated factors}

Subsequently, we analysed the rate of non-regression between SVR24 and SVR96 and associated factors. As shown in Figure 2, as the fibrosis stage increased at SVR24, the rate of non-regression at 96 weeks after DAA therapy increased. Among patients with stage F4 at SVR24, 58\% (7/12) showed non-regression of liver fibrosis stage based on LSM, compared with 15\% (17/110) for all patients.

As shown in Table 4, subsequent univariate analyses revealed that liver fibrosis stage at SVR 24 (F0-2 vs. $3-4, P<0.001)$, platelet count at SVR24 $(P=0.004)$, history of hypertension $(P=0.006)$, AST level at SVR24 $(P=0.003)$, YGTP level at SVR24 $(P=0.019)$, FIB-4 index at SVR24 $(P<0.001)$, and post-treatment Ang2 level $(P=0.001)$ were significantly associated with non-regression of liver fibrosis stage between SVR24 and SVR96. Subsequently, we conducted a multivariate logistic regression analysis using significant factors $(P \leq 0.001)$ in the univariate analysis (i.e., liver fibrosis stage, FIB-4 index, and Ang2 levels). As shown in Table 4, fibrosis stage at SVR24 (odds ratio 12.35, 95\% confidence interval, 3.8639.6; $P<0.001$ ) alone was significantly associated with non-regression between SVR24 and SVR96.

Next, we conducted a subgroup analysis of patients with liver fibrosis stage F3/4 at SVR24 (Table 5). A high Ang2 level post-treatment and high CAP value at SVR24 were significantly associated with nonregression of liver fibrosis stage between SVR24 and SVR96.

\section{Discussion}

DAAs are revolutionary anti-HCV drugs. Numerous patients have experienced successful HCV eradication in the past several years due to these novel DAAs. However, recent studies have revealed that even after successful HCV eradication by DAAs, the occurrence of HCC and deterioration of liver function are 
sometimes observed. The deterioration of liver fibrosis after HCV eradication is closely associated with $\mathrm{HCC}$ occurrence ${ }^{11}$ and causes the deterioration of liver function; thus, the regression of liver fibrosis after successful HCV eradication is a clinically important issue. Previously, we reported that $25 \%(29 / 116)$ of patients show non-regression of LSM-based liver fibrosis stage at 24 weeks after DAA completion. ${ }^{13}$ In this study, $11 \%$ of patients $(12 / 110)$ showed non-regression of LSM-based fibrosis stage at 96 weeks after the completion of DAAs. Thus, over a longer observation period, more patients with successful HCV eradication by DAAs could experience regression of the LSM-based fibrosis stage. A multivariate regression analysis revealed that baseline fibrosis stage and Ang2 levels were significantly associated with non-regression at 96 weeks after the completion of DAAs. These results are consistent with those of our shorter observational study (spanning 24 weeks) showing that the baseline fibrosis stage and Ang2 levels are significantly determinants of non-regression after DAA therapy. ${ }^{13}$

In addition, the observed association between an advanced fibrosis stage (F3/4) at baseline and nonregression of liver fibrosis after anti-HCV therapy is consistent with the results of a previous study in the IFN era. ${ }^{11}$ In a subgroup analysis of patients with advanced fibrosis (F3/4) at baseline (Table 3), we further showed that a high Ang2 level at baseline is significantly associated with non-regression of liver fibrosis stage at 96 weeks after DAA completion. Thus, high baseline Ang2 levels might be an important predictive factor for non-regression, even for long time periods (96 weeks) after DAA completion.

Because LSM could be affected by liver inflammation due to HCV infection, we analysed the period between 24 and 96 weeks after DAA completion (Table 4). Although a multivariate analysis revealed that advanced liver fibrosis stage (F0-2 vs. F3/4) alone was associated with non-regression, a subgroup analysis of patients with F3/4 at SVR24 revealed that high CAP and Ang2 values post-treatment were significantly associated with non-regression of liver fibrosis stage (Table 5). CAP is a well-established surrogate marker of liver steatosis. ${ }^{24}$ Liver steatosis is a risk factor for the development of liver fibrosis. ${ }^{25}$ Thus, even after successful HCV eradication, patients with liver steatosis should be carefully followed up and should take measures to improve liver steatosis. Elevated post-treatment Ang2 levels were also significantly associated with non-regression between 24 weeks and 96 weeks after the completion of DAA therapy in patients with F3/4 at SVR24 points, similar to the results obtained for the comparison between baseline and SVR96. Thus, serum Ang2 levels are a potential predictive marker of non-regression of liver fibrosis, even long after the completion of DAAs.

The ANG-TIE2 pathway has a unique effect on vascular stability. Ang1 is mainly expressed in mesenchymal cells and has agonistic effects on Tie2-mediated signalling, resulting in vessel stabilization and endothelial barrier function. ${ }^{15,16}$ Ang2 is mainly expressed in endothelial cells and is increased by VEGF, TGF, and hypoxia. ${ }^{26}$ In addition, it has been reported that portal hypertension-induced slow blood flow causes increased Ang2 expression. ${ }^{19,20}$ Ang2 has an antagonistic effect on Tie2-mediated signalling, resulting in the inhibition of ANG1-TIE2-mediated signalling, causing vascular instability, leakage, and inflammation. ${ }^{26}$ 
Several studies have shown that elevated serum Ang2 is a candidate biomarker in various liver diseases. Increased Ang2 expression in liver tissues is associated with the occurrence and recurrence of HCC after DAA treatment for hepatitis $C .{ }^{20}$ In this previous study, importantly, serum Ang2 levels after DAA therapy were significantly associated with liver tissue Ang2 expression levels. In addition, Lefere et al. reported the possibility that serum Ang2 levels could distinguish patients with NASH from those with simple liver steatosis. ${ }^{27}$ Mauro et al. reported that elevated serum Ang2 is associated with mortality and kidney outcomes in patients with decompensated cirrhosis with acute kidney injury. ${ }^{17}$ In addition, we have previously shown that baseline elevated Ang2 levels could predict non-regression of liver fibrosis stage based on LSM at 24 weeks after DAA treatment in patients with HCV infection. In this study, we showed that this association persists over a longer period, even at 96 weeks after DAA treatment in patients with HCV infection. Thus, elevated Ang2 could persist for a long duration and might have a pathogenic effect on liver fibrosis. It has been reported that elevated Ang2 expression causes vascular leakage and inflammation and might promote the progression of liver fibrosis. ${ }^{28}$

Recently, Ang2 has been identified as a potential therapeutic target ${ }^{26}$ in cancer and ophthalmologic diseases. ${ }^{26}$ Recently, the effectiveness of the inhibition of both Ang2 and VEGF in diabetic macular oedema has been reported ${ }^{29}$. Similarly, in liver disease, anti-Ang2 therapy is a potential novel therapeutic option. Lefere et al. observed higher serum Ang2 levels in patients with NASH than in patients with simple liver steatosis, ${ }^{27}$ and the inhibition of Ang2 restored liver fibrosis in a NASH mouse model. In addition, Pauta et al. reported that in a liver fibrosis rat model induced by $\mathrm{CCl} 4$, liver fibrosis and liver inflammation were reduced by the administration of an anti-angiopoietin 2 antibody. ${ }^{28}$

Accordingly, there is substantial evidence that the inhibition of Ang2 could be a novel therapeutic strategy in liver disease. In the present study, patients with elevated serum Ang2 levels showed non-regression of liver fibrosis, suggesting that anti-Ang2 therapy might lead to regression.

This study had several limitations. It was a retrospective study, and the number of patients was relatively small. Thus, a prospective, large-scale, and multicentre study is required to validate the findings. In addition, we evaluated the liver fibrosis stage using LSM. The gold standard for staging is liver biopsy; however, this procedure is invasive and carries a risk of sampling error. Thus, we utilized the LSM-based liver fibrosis stage, and this should be considered when interpreting the study results.

In conclusion, an advanced liver fibrosis stage and baseline high serum Ang2 levels are associated with non-regression of liver fibrosis stage at 96 weeks after the completion of DAA therapy. Thus, careful monitoring is necessary in these patients, even after successful HCV eradication by DAAs.

\section{Declarations}

\section{Data availability}


The datasets generated during and/or analysed during the current study are available from the corresponding author on reasonable request.

\section{Acknowledgments}

We thank the patients who participated in this study and their families. This study was supported in part by grants from the Japan Agency for Medical Research and Development (AMED; grant nos. JP20fk0210072, JP20fk0210064, JP20fk0210056, JP20fk0310101, JP20fk0210047, JP20fk0210048, JP20fk0210058, JP20fk0210066, and JP20fk0210067) and SPS KAKENHI (grant number 19K08458).

\section{Author contributions}

N.K. and G.S. designed this study, performed the statistical analyses, and wrote the manuscript. M.K., O.M., Y.T., K.S., A.K., M.N., T.S., Y.K., R.Y., T.K., T.S., and M.N. collected the data. M. Natsuizaka, K.M., and K.O. provided hepatological advice and edited the manuscript. N.S. revised the manuscript for important intellectual content.

\section{ADDITIONAL INFORMATION}

Competing interests: Professor Naoya Sakamoto received lecture fees from Bristol Myers Squibb and Pharmaceutical K. K, grants and endowments from MSD K. K and Chugai Pharmaceutical Co., Ltd, and a research grant from Gilead Sciences Inc. Dr. Goki Suda received research grants from Bristol Myers Squibb. The other authors declare no competing interests.

Clinical trial number: UMIN000031091

\section{References}

1. Suda, G. et al. Retreatment with sofosbuvir, ledipasvir, and add-on ribavirin for patients who failed daclatasvir and asunaprevir combination therapy. J Gastroenterol, doi:10.1007/s00535-017-1328-z (2017).

2. Suda, G., Ogawa, K., Morikawa, K. \& Sakamoto, N. Treatment of hepatitis C in special populations. J Gastroentero/ 53, 591-605, doi:10.1007/s00535-017-1427-x (2018).

3. Suda, G. et al. Safety and efficacy of elbasvir and grazoprevir in Japanese hemodialysis patients with genotype $1 \mathrm{~b}$ hepatitis C virus infection. J Gastroentero/ 54, 78-86, doi:10.1007/s00535-0181495-6 (2019).

4. Suda, G. et al. Efficacy and safety of daclatasvir and asunaprevir combination therapy in chronic hemodialysis patients with chronic hepatitis C. J Gastroenterol, doi:10.1007/s00535-016-1162-8 (2016).

5. Suda, G. et al. Safety and efficacy of glecaprevir and pibrentasvir in Japanese hemodialysis patients with genotype 2 hepatitis C virus infection. J Gastroentero/ 54, 641-649, doi:10.1007/s00535-01901556-y (2019). 
6. Suda, G. et al. Daclatasvir and asunaprevir in hemodialysis patients with hepatitis $C$ virus infection: a nationwide retrospective study in Japan. J Gastroentero/ 53, 119-128, doi:10.1007/s00535-017-1353y (2018).

7. Suda, G. \& Sakamoto, N. Recent advances in the treatment of hepatitis $C$ virus infection for special populations and remaining problems. J Gastroenterol Hepatol, doi:10.1111/jgh.15189 (2020).

8. Rockstroh, J. K. et al. Efficacy and Safety of Glecaprevir/Pibrentasvir in Patients Coinfected With Hepatitis $C$ Virus and Human Immunodeficiency Virus Type 1: The EXPEDITION-2 Study. Clin Infect Dis 67, 1010-1017, doi:10.1093/cid/ciy220 (2018).

9. Naggie, S. et al. Ledipasvir and Sofosbuvir for HCV in Patients Coinfected with HIV-1. N Engl J Med 373, 705-713, doi:10.1056/NEJMoa1501315 (2015).

10. Agarwal, K. et al. Sofosbuvir/velpatasvir for 12weeks in genotype 1-4 HCV-infected liver transplant recipients. J Hepato/ 69, 603-607, doi:10.1016/j.jhep.2018.05.039 (2018).

11. Tachi, Y. et al. Progressive fibrosis significantly correlates with hepatocellular carcinoma in patients with a sustained virological response. Hepatol Res 45, 238-246, doi:10.1111/hepr.12331 (2015).

12. Shiratori, Y. et al. Histologic improvement of fibrosis in patients with hepatitis $C$ who have sustained response to interferon therapy. Ann Intern Med 132, 517-524, doi:10.7326/0003-4819-132-7200004040-00002 (2000).

13. Kawagishi, N. et al. High serum angiopoietin-2 level predicts non-regression of liver stiffness measurement-based liver fibrosis stage after direct-acting antiviral therapy for hepatitis C. Hepatol Res 50, 671-681, doi:10.1111/hepr.13490 (2020).

14. Mauro, E. et al. Portal pressure and liver stiffness measurements in the prediction of fibrosis regression after sustained virological response in recurrent hepatitis C. Hepatology 67, 1683-1694, doi:10.1002/hep.29557 (2018).

15. Souma, T. et al. Context-dependent functions of angiopoietin 2 are determined by the endothelial phosphatase VEPTP. Proc Natl Acad Sci U S A 115, 1298-1303, doi:10.1073/pnas.1714446115 (2018).

16. Benest, A. V. et al. Angiopoietin-2 is critical for cytokine-induced vascular leakage. PLoS One 8, e70459, doi:10.1371/journal.pone.0070459 (2013).

17. Allegretti, A. S. et al. Serum Angiopoietin-2 Predicts Mortality and Kidney Outcomes in Decompensated Cirrhosis. Hepatology 69, 729-741, doi:10.1002/hep.30230 (2019).

18. Shigesawa, T. et al. Baseline angiopoietin-2 and FGF19 levels predict treatment response in patients receiving multikinase inhibitors for hepatocellular carcinoma. JGH Open 4, 880-888, doi:10.1002/jgh3.12339 (2020).

19. Goettsch, W. et al. Flow-dependent regulation of angiopoietin-2. J Cell Physio/ 214, 491-503, doi:10.1002/jcp.21229 (2008).

20. Faillaci, F. et al. Liver Angiopoietin-2 Is a Key Predictor of De Novo or Recurrent Hepatocellular Cancer After Hepatitis C Virus Direct-Acting Antivirals. Hepatology 68, 1010-1024, doi:10.1002/hep.29911 (2018). 
21. Seko, Y. et al. Presence of varices in patients after hepatitis $C$ virus eradication predicts deterioration in the FIB-4 index. Hepatol Res 49, 473-478, doi:10.1111/hepr.13296 (2019).

22. Kawagishi, N. et al. Liver steatosis and dyslipidemia after HCV eradication by direct acting antiviral agents are synergistic risks of atherosclerosis. PLoS One 13, e0209615, doi:10.1371/journal.pone.0209615 (2018).

23. Castera, L. et al. Prospective comparison of transient elastography, Fibrotest, APRI, and liver biopsy for the assessment of fibrosis in chronic hepatitis C. Gastroenterology 128, 343-350 (2005).

24. Karlas, T. et al. Individual patient data meta-analysis of controlled attenuation parameter (CAP) technology for assessing steatosis. J Hepato/ 66, 1022-1030, doi:10.1016/j.jhep.2016.12.022 (2017).

25. Parthasarathy, G., Revelo, X. \& Malhi, H. Pathogenesis of Nonalcoholic Steatohepatitis: An Overview. Hepatol Commun 4, 478-492, doi:10.1002/hep4.1479 (2020).

26. Saharinen, P., Eklund, L. \& Alitalo, K. Therapeutic targeting of the angiopoietin-TIE pathway. Nat Rev Drug Discov 16, 635-661, doi:10.1038/nrd.2016.278 (2017).

27. Lefere, S. et al. Angiopoietin-2 Promotes Pathological Angiogenesis and Is a Therapeutic Target in Murine Nonalcoholic Fatty Liver Disease. Hepatology 69, 1087-1104, doi:10.1002/hep.30294 (2019).

28. Pauta, M. et al. Overexpression of angiopoietin-2 in rats and patients with liver fibrosis. Therapeutic consequences of its inhibition. Liver Int 35, 1383-1392, doi:10.1111/liv.12505 (2015).

29. Sahni, J. et al. Simultaneous Inhibition of Angiopoietin-2 and Vascular Endothelial Growth Factor-A with Faricimab in Diabetic Macular Edema: BOULEVARD Phase 2 Randomized Trial. Ophthalmology 126, 1155-1170, doi:10.1016/j.ophtha.2019.03.023 (2019).

\section{Tables}

Table 1. Baseline characteristics of patients 


\begin{tabular}{|c|c|c|c|c|}
\hline & All & F0-2 & F3-4 & $\begin{array}{l}P \\
\text { value }\end{array}$ \\
\hline Number & 110 & 81 & 29 & \\
\hline Age $\left(\right.$ years) ${ }^{\mathrm{a}}$ & $66(22-87)$ & $64(22-83)$ & $66(44-87)$ & 0.144 \\
\hline Sex (male/female) & $41 / 69$ & $29 / 52$ & $12 / 17$ & 0.594 \\
\hline $\begin{array}{l}\text { DCV/ASV, SOF/LDV, SOF/RBV, } \\
\text { OBV/PTV/r }\end{array}$ & $19 / 47 / 39 / 5$ & $10 / 38 / 29 / 4$ & $9 / 9 / 10 / 1$ & 0.128 \\
\hline HCV-RNA $(\log I U / m L)^{a}$ & $6.3(4.2-7.2)$ & $6.3(4.2-7.2)$ & $6.3(4.7-7.2)$ & 0.757 \\
\hline $\operatorname{BMI}\left(\mathrm{kg} / \mathrm{m}^{2}\right)^{\mathrm{a}}$ & $22(15.8-36.3)$ & $21.8(15.8-31)$ & $\begin{array}{l}22.5(17.1- \\
36.3)\end{array}$ & 0.238 \\
\hline $\mathrm{F} 0-1 / 2 / 3 / 4$ & $62 / 19 / 8 / 21$ & $62 / 19 / 0 / 0$ & $0 / 0 / 8 / 21$ & \\
\hline Platelet count $\left(\times 10^{4}\right)^{a}$ & $16.2(2.6-37.3)$ & $17.8(2.6-37.3)$ & $11.4(5.4-24.7)$ & $\begin{array}{l}\star \\
<0.001\end{array}$ \\
\hline AST $(I U / L)^{a}$ & $39(16-180)$ & $34(16-180)$ & $57(33-125)$ & $\begin{array}{l}* \\
<0.001\end{array}$ \\
\hline $\operatorname{ALT}(I U / L)^{a}$ & $38(6-273)$ & $31(6-273)$ & $58(22-101)$ & $\begin{array}{l}* \\
<0.001\end{array}$ \\
\hline yGTP $(I U / L)^{a}$ & $29(9-559)$ & $24(9-276)$ & $40(14-559)$ & $\begin{array}{l}* \\
<0.001\end{array}$ \\
\hline FIB-4 index ${ }^{a}$ & $\begin{array}{l}2.79(0.54- \\
13.51)\end{array}$ & $\begin{array}{l}2.41(0.54- \\
13.51)\end{array}$ & $\begin{array}{l}5.41(1.55- \\
8.69)\end{array}$ & $\begin{array}{l}* \\
<0.001\end{array}$ \\
\hline Angiopoietin-2 $(\mathrm{pg} / \mathrm{mL})^{a}$ & $\begin{array}{l}305.6(131.9- \\
899.9)\end{array}$ & $\begin{array}{l}289.5(131.9- \\
864.5)\end{array}$ & $\begin{array}{l}434.1(155.5- \\
899.9)\end{array}$ & $\star 0.01$ \\
\hline $\operatorname{CAP}(\mathrm{dB} / \mathrm{m})^{a}$ & $214(100-386)$ & $210(100-343)$ & $226(106-386)$ & 0.286 \\
\hline Diabetes mellitus ( $n, \%)$ & $22(20 \%)$ & $13(16 \%)$ & $9(31 \%)$ & 0.083 \\
\hline High blood pressure (n, \%) & $38(35 \%)$ & $21(30 \%)$ & $17(59 \%)$ & $\star 0.001$ \\
\hline Dyslipidaemia (n, \%) & $20(18 \%)$ & $14(17 \%)$ & $6(21 \%)$ & 0.683 \\
\hline
\end{tabular}

Abbreviations: $\mathrm{HCV}$, hepatitis C virus; $\mathrm{BMI}$, body mass index; $\mathrm{AST}$, aspartate aminotransferase; ALT, alanine aminotransferase; $ү$ GTP, $\gamma$-glutamyl transpeptidase; FIB-4, fibrosis 4; CAP, Controlled Attenuation Parameter.

aData are shown as median (range) values.

*Statistically significant difference, $\mathrm{P}<0.05$ 
Table 2. Factors associated with non-regression of liver fibrosis stage based on LSM between baseline and SVR96 in HCV-infected patients treated with DAAs

\begin{tabular}{|c|c|c|c|c|c|}
\hline & Regression & $\begin{array}{l}\text { Non- } \\
\text { regression }\end{array}$ & $\begin{array}{l}\text { Univariate } \\
\text { analysis }\end{array}$ & $\begin{array}{l}\text { Multivariate } \\
\text { analysis }\end{array}$ & $\begin{array}{l}\text { Odds } \\
\text { ratio }\end{array}$ \\
\hline Number & 98 & 12 & & & \\
\hline Age (years) ${ }^{a}$ & $66(22-87)$ & $65(56-75)$ & 0.943 & & \\
\hline Sex (male/female) & $38 / 60$ & $3 / 9$ & 0.529 & & \\
\hline $\begin{array}{l}\text { DCV/ASV, SOF/LDV } \\
\text { SOF/RBV, OBV/PTV/r }\end{array}$ & $16 / 43 / 35 / 4$ & $3 / 4 / 4 / 1$ & 0.756 & & \\
\hline HCV-RNA $(\log I U / m L)^{a}$ & $\begin{array}{l}6.4(4.2- \\
7.2)\end{array}$ & $\begin{array}{l}5.5(4.4- \\
7.1)\end{array}$ & $\star 0.025$ & & \\
\hline $\mathrm{BMI}\left(\mathrm{kg} / \mathrm{m}^{2}\right)^{\mathrm{a}}$ & $\begin{array}{l}21.9(15.8- \\
36.3)\end{array}$ & $\begin{array}{l}22.5(15.8- \\
31.2)\end{array}$ & 0.934 & & \\
\hline$F 0-2 / 3-4$ & $77 / 21$ & $4 / 8$ & $\star 0.002$ & 0.033 & $\begin{array}{l}4.56 \\
(1.13- \\
18.3)\end{array}$ \\
\hline Platelet count $\left(\times 10^{4}\right)^{a}$ & $\begin{array}{l}16.8(2.6- \\
37.3)\end{array}$ & $\begin{array}{l}12.7(5.2- \\
24.7)\end{array}$ & 0.085 & & \\
\hline AST $(I U / L)^{a}$ & $\begin{array}{l}38(16- \\
180)\end{array}$ & $\begin{array}{l}50(32- \\
125)\end{array}$ & $\star 0.029$ & & \\
\hline $\operatorname{ALT}(I U / L)^{a}$ & $38(6-273)$ & $\begin{array}{l}33(18- \\
101)\end{array}$ & 0.814 & & \\
\hline yGTP (IU/L)a & $\begin{array}{l}27.5(9- \\
276)\end{array}$ & $\begin{array}{l}39(14- \\
559)\end{array}$ & 0.141 & & \\
\hline FIB-4 index ${ }^{a}$ & $\begin{array}{l}2.73(0.54- \\
13.51)\end{array}$ & $\begin{array}{l}4.13(2.18- \\
8.69)\end{array}$ & $\star 0.005$ & 0.37 & \\
\hline Angiopoietin-2 $(\mathrm{pg} / \mathrm{mL})^{a}$ & $\begin{array}{l}297.8 \\
(131.9- \\
864.5)\end{array}$ & $\begin{array}{l}461.5 \\
(220.4- \\
899.9)\end{array}$ & $\star 0.004$ & 0.039 & $\begin{array}{l}1.004 \\
(1.0- \\
1.01)\end{array}$ \\
\hline $\operatorname{CAP}(\mathrm{dB} / \mathrm{m})^{\mathrm{a}}$ & $\begin{array}{l}213(100- \\
386)\end{array}$ & $\begin{array}{l}221(100- \\
241)\end{array}$ & 0.773 & & \\
\hline Diabetes mellitus ( $n, \%)$ & $18(18 \%)$ & $4(33 \%)$ & 0.195 & & \\
\hline High blood pressure $(n, \%)$ & $31(32 \%)$ & $7(58 \%)$ & 0.068 & & \\
\hline Dyslipidaemia (n, \%) & 19 (19\%) & $1(8 \%)$ & 0.314 & & \\
\hline
\end{tabular}

Abbreviations: HCV, hepatitis C virus; BMI, body mass index; AST, aspartate aminotransferase; ALT, alanine aminotransferase; $ү$ GTP, $\gamma$-glutamyl transpeptidase; FIB-4, fibrosis 4; CAP, Controlled Attenuation 
Parameter

${ }^{a}$ Data are shown as median (range) values.

*Statistically significant difference, $\mathrm{P}<0.05$

Table 3. Factors associated with non-regression of liver fibrosis stage based on LSM between baseline and SVR96 in HCV-infected patients with baseline advanced liver fibrosis, who were treated with DAAs

\begin{tabular}{|c|c|c|c|}
\hline & Regression & Non-regression & $P$-value \\
\hline Number & 21 & 8 & \\
\hline Age $\left(\right.$ years) ${ }^{a}$ & $66(44-87)$ & $67(58-72)$ & 0.943 \\
\hline Sex (male/female) & $10 / 11$ & $2 / 6$ & 0.408 \\
\hline DCV/ASV, SOF/LDV, SOF/RBV, OBV/PTV/r & $7 / 6 / 7 / 1$ & $2 / 3 / 3 / 0$ & 0.876 \\
\hline HCV-RNA $(\log I U / m L)^{a}$ & $6.4(4.8-7.2)$ & $6(4.7-7.1)$ & $\star 0.025$ \\
\hline $\mathrm{BMI}\left(\mathrm{kg} / \mathrm{m}^{2}\right)^{\mathrm{a}}$ & $22.2(17.1-36.3)$ & $22.6(20-31.2)$ & 0.582 \\
\hline F3/4 & $7 / 14$ & $1 / 7$ & 0.381 \\
\hline Platelet count $\left(\times 10^{4}\right)^{a}$ & $11.4(5.4-19.3)$ & $11.9(7.7-24.7)$ & 0.72 \\
\hline AST (IU/L) ${ }^{a}$ & $54(33-122)$ & $63(39-125)$ & 0.324 \\
\hline $\operatorname{ALT}(I U / L)^{a}$ & $56(25-96)$ & $61(22-101)$ & 0.72 \\
\hline yGTP $(I U / L)^{a}$ & $37(17-105)$ & $81(14-559)$ & 0.237 \\
\hline FIB-4 index ${ }^{a}$ & $5.41(1.55-8.37)$ & $5.57(2.18-8.69)$ & 0.487 \\
\hline Angiopoietin-2 $(\mathrm{pg} / \mathrm{mL})^{a}$ & 344.1 (155.5-848) & $493(395.9-899.9)$ & *0.024 \\
\hline $\operatorname{CAP}(\mathrm{dB} / \mathrm{m})^{a}$ & $224(106-386)$ & $235(198-241)$ & 0.374 \\
\hline Diabetes mellitus ( $\mathrm{n}, \%)$ & $5(24 \%)$ & $4(50 \%)$ & 0.18 \\
\hline High blood pressure $(\mathrm{n}, \%)$ & $12(57 \%)$ & $5(63 \%)$ & 0.568 \\
\hline Dyslipidaemia (n, \%) & $5(24 \%)$ & $1(13 \%)$ & 0.457 \\
\hline
\end{tabular}

Abbreviations: HCV, hepatitis C virus; BMI, body mass index; AST, aspartate aminotransferase; ALT, alanine aminotransferase; yGTP, y-glutamyl transpeptidase; FIB-4, fibrosis 4; CAP, Controlled Attenuation Parameter

${ }^{a}$ Data are shown as median (range) values. 
$\star P<0.05$

Table 4. Factors associated with non-regression of liver fibrosis stage based on LSM between SVR24 and SVR96 in HCV-infected patients treated with DAAs

\begin{tabular}{|c|c|c|c|c|c|}
\hline & Regression & $\begin{array}{l}\text { Non- } \\
\text { regression }\end{array}$ & $\begin{array}{l}\text { Univariate } \\
\text { analysis }\end{array}$ & $\begin{array}{l}\text { Multivariate } \\
\text { analysis }\end{array}$ & Odds ratio \\
\hline Number & 93 & 17 & & & \\
\hline Age (years) ${ }^{a}$ & $67(23-84)$ & $67(57-88)$ & 0.305 & & \\
\hline Sex (male/female) & $36 / 57$ & $5 / 12$ & 0.466 & & \\
\hline $\begin{array}{l}\text { DCV/ASV, SOF/LDV, } \\
\text { SOF/RBV, OBV/PTV/r }\end{array}$ & $14 / 42 / 33 / 4$ & $5 / 5 / 6 / 1$ & 0.452 & & \\
\hline HCV-RNA $(\log \mathrm{IU} / \mathrm{mL})^{\mathrm{a}}$ & N.D & N.D & - & & \\
\hline Baseline BMI $\left(\mathrm{kg} / \mathrm{m}^{2}\right)^{a}$ & $\begin{array}{l}22(15.8- \\
36.3)\end{array}$ & $\begin{array}{l}22.5(15.8- \\
31.2)\end{array}$ & 0.828 & & \\
\hline F0-2/3-4 at SVR24 & $81 / 12$ & $6 / 11$ & $\star<0.001$ & $<0.001$ & $\begin{array}{l}12.35(3.86- \\
39.6)\end{array}$ \\
\hline $\begin{array}{l}\text { Platelet count }\left(\times 10^{4}\right)^{a} \text { at } \\
\text { SVR24 }\end{array}$ & $\begin{array}{l}17.8(3.6- \\
34.1)\end{array}$ & $\begin{array}{l}13.8(4.8- \\
24.3)\end{array}$ & $\star 0.004$ & & \\
\hline AST (IU/L) ${ }^{a}$ at SVR24 & $21(11-50$ & $26(17-128)$ & $\star 0.003$ & & \\
\hline ALT (IU/L) a at SVR24 & $14(5-50)$ & $16(9-121)$ & 0.104 & & \\
\hline yGTP $(I U / L)^{a}$ at SVR24 & 16(9-92) & $22(8-865)$ & $\star 0.019$ & & \\
\hline FIB-4 index ${ }^{a}$ at SVR24 & $\begin{array}{l}2.07(0.5- \\
9.35)\end{array}$ & $\begin{array}{l}3.32(1.92- \\
9.01)\end{array}$ & $*<0.001$ & 0.107 & \\
\hline $\begin{array}{l}\text { Post-treatment } \\
\text { Angiopoietin-2 }(\mathrm{pg} / \mathrm{mL})^{\text {a }}\end{array}$ & $\begin{array}{l}266(60.2- \\
774.4)\end{array}$ & $\begin{array}{l}416.6(219.1- \\
913)\end{array}$ & $\star 0.001$ & 0.088 & \\
\hline $\mathrm{CAP}(\mathrm{dB} / \mathrm{m})^{\text {a }}$ at SVR24 & $\begin{array}{l}219(122- \\
385)\end{array}$ & $\begin{array}{l}228(145- \\
301)\end{array}$ & 0.761 & & \\
\hline Diabetes mellitus ( $\mathrm{n}, \%)$ & $17(18 \%)$ & $5(29 \%)$ & 0.228 & & \\
\hline $\begin{array}{l}\text { High blood pressure (n, } \\
\%)\end{array}$ & $27(29 \%)$ & $11(65 \%)$ & $\star 0.006$ & & \\
\hline Dyslipidaemia $(\mathrm{n}, \%)$ & $19(14 \%)$ & $1(6 \%)$ & 0.135 & & \\
\hline
\end{tabular}

Abbreviations: HCV, hepatitis C virus; BMI, body mass index; AST, aspartate aminotransferase; ALT, alanine aminotransferase; $\gamma$ GTP, $\gamma$-glutamyl transpeptidase; FIB-4, fibrosis 4; CAP, Controlled Attenuation Parameter. ${ }^{\text {aD }}$ ata are shown as median (range) values. ${ }^{*}$ Statistically significant difference, $\mathrm{P}<0.05$ 
Table 5 . Factors associated with non-regression of liver fibrosis stage based on LSM between SVR24 and SVR96 in HCV infected patients with advanced liver fibrosis at SVR24, who were treated with DAAs

\begin{tabular}{|c|c|c|c|}
\hline & Regression & Non-regression & $P$-value \\
\hline Number & 12 & 11 & \\
\hline Age $\left(\right.$ years) ${ }^{a}$ & $69(44-80)$ & $66(57-88)$ & 0.608 \\
\hline Sex (male/female) & $6 / 6$ & $3 / 8$ & 0.4 \\
\hline DCV/ASV, SOF/LDV, SOF/RBV, OBV/PTV/r & $5 / 3 / 3 / 1$ & $3 / 3 / 5 / 0$ & 0.581 \\
\hline HCV-RNA $(\log \mathrm{IU} / \mathrm{mL})^{a}$ & N.D & N.D & - \\
\hline Baseline BMI $\left(\mathrm{kg} / \mathrm{m}^{2}\right)^{\mathrm{a}}$ & 21.6(18.3-28.8) & $22.5(20-31.2)$ & 0.923 \\
\hline F0-2/3-4 at SVR24 & $7 / 5$ & $4 / 7$ & 0.292 \\
\hline Platelet count $\left(\times 10^{4}\right)^{a}$ at SVR24 & 11.6(7.1-19.4) & $11.1(4.8-24.3)$ & 0.928 \\
\hline AST (IU/L) ${ }^{\text {a }}$ at SVR24 & $25(15-50)$ & $26(18-128)$ & 0.525 \\
\hline $\operatorname{ALT}(I U / L){ }^{a}$ at SVR24 & $21(8-50)$ & $23(9-121)$ & 0.487 \\
\hline yGTP $(I U / L)^{a}$ at SVR24 & $24(12-65)$ & $22(8-885)$ & 0.740 \\
\hline FIB-4 index ${ }^{a}$ at SVR24 & $3.48(1.99-5.17)$ & $4.07(1.92-9.01)$ & 0.316 \\
\hline Post-treatment Angiopoietin-2 $(\mathrm{pg} / \mathrm{mL})^{a}$ & $388(258-620)$ & $544(351-913)$ & $\star 0.044$ \\
\hline $\mathrm{CAP}(\mathrm{dB} / \mathrm{m}){ }^{a}$ at SVR24 & $216(122-286)$ & 262(201-301) & $\star 0.037$ \\
\hline Diabetes mellitus ( $\mathrm{n}, \%)$ & $2(17 \%)$ & $5(45 \%)$ & 0.148 \\
\hline High blood pressure $(\mathrm{n}, \%)$ & $5(42 \%)$ & $7(64 \%)$ & 0.292 \\
\hline Dyslipidaemia (n, \%) & $2(17 \%)$ & $1(9 \%)$ & 0.534 \\
\hline
\end{tabular}

Abbreviations: $\mathrm{HCV}$, hepatitis C virus; BMI, body mass index; AST, aspartate aminotransferase; ALT, alanine aminotransferase; $ү$ GTP, $y$-glutamyl transpeptidase; FIB-4, fibrosis 4; CAP, Controlled Attenuation Parameter

${ }^{a}$ Data are shown as median (range) values.

*Statistically significant difference, $P<0.05$

\section{Figures}




\begin{tabular}{llllll}
\hline F0-1 & F2 & F3 & F4 & Regressed & Non-regression \\
\hline n & & & n/n (\%) \\
\hline
\end{tabular}

\section{Baseline}

$\begin{array}{lllllll}\text { F0-1 } & 60 & 2 & 0 & 0 & 60 / 62(97) & 2 / 62(3) \\ \text { F2 } & 17 & 0 & 2 & 0 & 17 / 19(89) & 2 / 19(11) \\ \text { F3 } & 3 & 4 & 1 & 0 & 7 / 8(87) & 1 / 8(13) \\ \text { F4 } & 5 & 3 & 6 & 7 & 14 / 21(67) & 7 / 21(33)\end{array}$

Total

$98 / 110(89) \quad 12 / 110(11)$

Figure 1

Changes in liver fibrosis stage based on LSM between baseline and SVR96 in HCV-infected patients treated with DAAs LSM, liver stiffness measurement; SVR, sustained virological response; DAAs, directacting antivirals The grey area represents regression of liver fibrosis after DAAs therapy.

At SVR96

Disease Activity

\begin{tabular}{llllll}
\hline F0-1 & F2 & F3 & F4 & Regressed & Non-regression \\
\hline $\mathbf{n}$ & & & n/n (\%) \\
\hline
\end{tabular}

\section{At SVR24}

F0-1

F2

F3

F4

\section{Total}

72

9

3

1

2

1

0

4

2
0

0

0

7
72/76 (95)

4/76 (5)

9/11 (82)

7/11 (64)

5/12 (42)
2/11 (18)

4/11 (36)

7/12 (58)

\section{Figure 2}

Changes in liver fibrosis stage based on LSM between SVR24 and SVR96 in HCV-infected patients treated with DAAs LSM, liver stiffness measurement; SVR, sustained virological response; DAAs, direct-acting antivirals The grey area represents the regression of liver fibrosis after DAA therapy. 


\section{Supplementary Files}

This is a list of supplementary files associated with this preprint. Click to download.

- supplementaryFig.docx 